\title{
Visualization of Extracellular Vesicles of Prokaryotes and Eukaryotic Microbes
}

*Correspondence to:

Kim KW,

(iD) http://orcid.org/0000-0002-7010-0336

Tel: +82-54-530-1246

Fax: +82-54-530-1248

E-mail:kiwoo@knu.ac.kr

Received December 19, 2018

Revised December 27, 2018

Accepted December 27, 2018

\author{
Ki Woo Kim ${ }^{1,2}$ * \\ ${ }^{1}$ School of Ecology and Environmental System, Kyungpook National University, Sangju 37224, Korea \\ '2Tree Diagnostic Center, Kyungpook National University, Sangju 37224, Korea
}

The release of nanoscale membrane-bound vesicles is common in all three domains of life. These vesicles are involved in a variety of biological processes such as cell-to-cell communication, horizontal gene transfer, and substrate transport. Prokaryotes including bacteria and archaea release membrane vesicles (MVs) (20 to $400 \mathrm{~nm}$ in diameter) into their extracellular milieu. In spite of structural differences in cell envelope, both Gram-positive and negative bacteria produce MVs that contain the cell membrane of each bacterial species. Archaeal MVs characteristically show surface-layer encircling the vesicles. Filamentous fungi and yeasts as eukaryotic microbes produce bilayered exosomes that have varying electron density. Microbes also form intracellular vesicles and minicells that are similar to MVs and exosomes in shape. Electron and fluorescence microscopy could reveal the presence of DNA in MVs and exosomes. Given the biogenesis of extracellular vesicles from the donor cell, in situ high-resolution microscopy can provide insights on the structural mechanisms underlying the formation and release of microbial extracellular vesicles.

Key Words: Archaea, Bacteria, Fungi, Membrane vesicle

\section{MICROBIAL EXTRACELLULAR VESICLES}

Cells release several types of vesicles into their extracellular environment (Raposo \& Stoorvogel, 2013). Such a release of spherical, membranous structures from cell surfaces is prevalent in organisms from all three domains of life (Deatherage \& Cookson, 2012). These are complex structures that are limited by a lipid bilayer and enclose soluble hydrophilic components derived from the cytosol of the donor cell (Thery et al., 2009). Depending on physicochemical characteristics and donor organisms, these vesicles are generally referred to as ectosomes, exosomes, microvesicles, microvesicles, microparticles, and others (Raposo \& Stoorvogel, 2013). The membranous vesicles from bacteria and archaea are commonly described as membrane vesicles (MVs), whereas those from fungi and mammals are called exosomes or shedding microvesicles (Deatherage \& Cookson, 2012).

Prokaryotic MVs mainly consist of the cell membrane and typically range from 20 to $400 \mathrm{~nm}$ in diameter (Fig. 1) (Joffe et al., 2016; Toyofuku et al., 2015). They allow the longdistance dissemination of prokaryotic products into the environment, inter-kingdom communication, maintenance of the biofilm structure, and horizontal gene transfer (Pérez-Cruz et al., 2015). Fungal exosomes are involved in the transport of proteins, lipids, polysaccharides, and pigments into their extracellular environment (Oliveira et al., 2010). This review aims to highlight microscopic views of extracellular vesicles of prokaryotes and eukaryotic microbes including archaea, mycoplasmas, and yeasts. It can provide clues to unanswered questions in microbial biology and pathogenicity in humans, domestic animals, and plants.

\section{GRAM-NEGATIVE BACTERIAL MVs}

MVs have been rigorously studied from Gram-negative bacteria since first observed in the 1960s (Knox et al., 1966). All

@ This is an open-access article distributed under the terms of the Creative Commons Attribution Non-Commercial License (http://creativecommons.org/licenses/by-nc/4.0) which permits unrestricted noncommercial use, distribution, and reproduction in any medium, provided the original work is properly cited.

Copyrights @ 2018 by Korean Society of Microscopy 

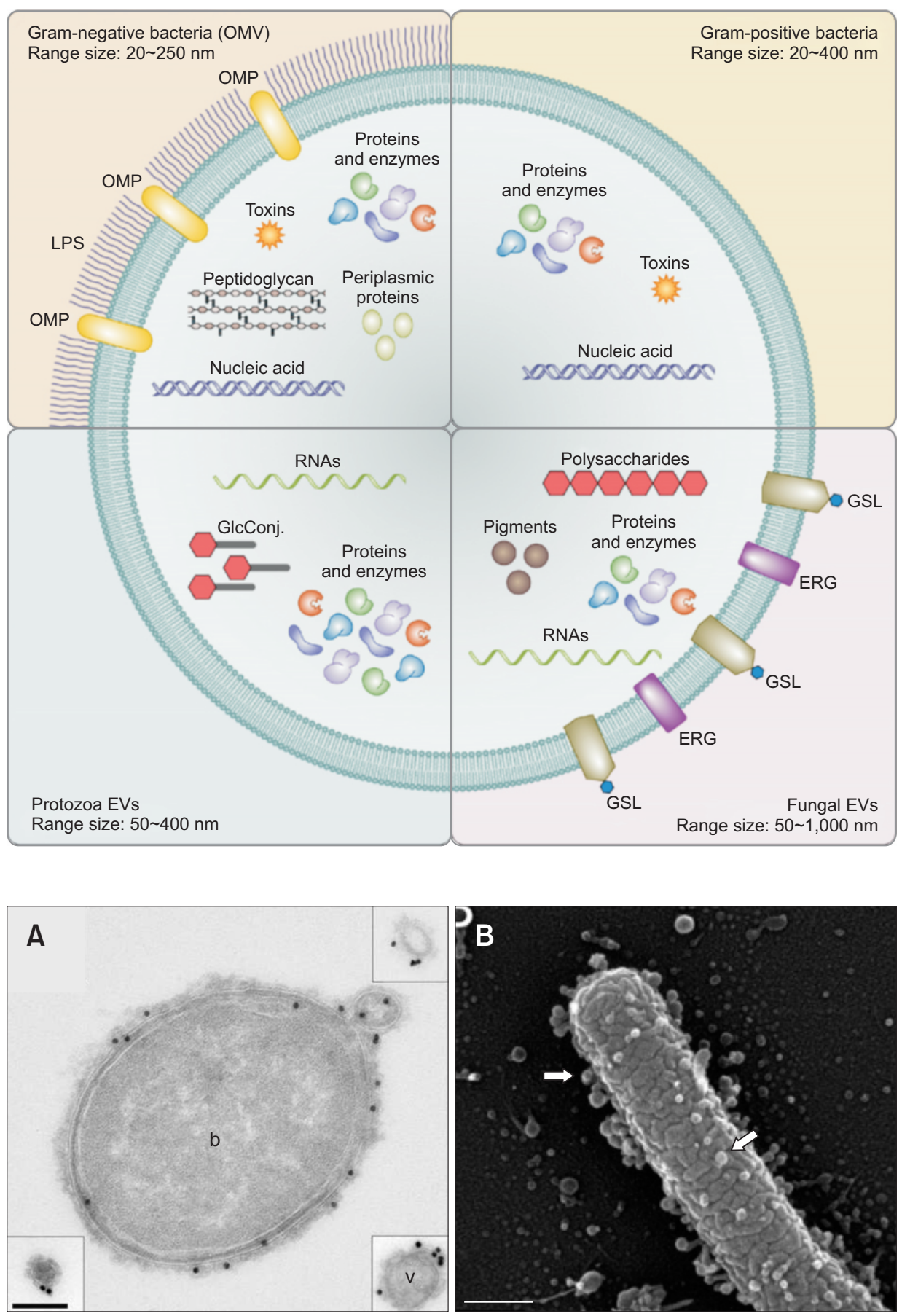

Fig. 1. Schematics of extracellular vesicles of microbial cells. ERG, ergosterol; GlcConj, glycoconjugates; GSL, glycosphingolipids; LPS, lipopolysaccharide; OMP, outer membrane protein. From Joffe et al., 2016 with permission from the publisher.

Fig. 2. Membrane vesicles of Gram-negative bacteria. (A) Transmission electron micrograph of Escherichia coli (b) labeled with anti-lipopolysaccharide antibody and Protein A Gold. Subsets depict membrane vesicles. Bar $=150 \mathrm{~nm}$. (B) Scanning electron micrograph of Xylella fastidiosa having membrane vesicles (arrows). Bar $=200 \mathrm{~nm}$. A: from Bielaszewska et al., 2017; B: from Ionescu et al., 2014 with permission from the publisher.

types of Gram-negative bacteria have been known to produce MVs, also referred to as outer MVs in case of Gram-negative bacteria, in a variety of environments including planktonic cultures, fresh and salt water, biofilms, inside eukaryotic cells, and within mammalian hosts (Schwechheimer \& Kuehn, 2015). For example, enterohemorrhagic Escherichia coli O157 produced MVs blebbing from the bacterial surface and liberated from the bacteria (Fig. 2A) (Bielaszewska et al., 2017). Immunogold labeling revealed the presence of lipopolysac- charide probably derived from the bacterial outer membrane on the vesicle surface.

Scanning electron microscopy unraveled MVs from the xylem-limited bacterium Xylella fastidiosa (Fig. 2B) (Ionescu et al., 2014). Some MVs were attached to cells, whereas others were unattached in planktonic samples. When their MVs were stained with DAPI and FM4-64 (lipophilic fluorescent dye), they showed fluorescence, indicating the presence of DNA and lipid in and on MVs (Ionescu et al., 2014). 
In addition, mycoplasmas (Class Mollicutes) are known to naturally produce MVs during in vitro culture (Chernov et al., 2014). Electron microscopy and atomic force microscopy revealed the presence of MVs from Acholeplasma laidlawii PG8 on the cell surface. They were spherical, bilayered, and ranged from 70 to $120 \mathrm{~nm}$ in diameter.

\section{GRAM-POSITIVE BACTERIAL MVs}

Although discovered 30 years later than their Gram-negative counterparts, Gram-positive bacterial MVs have been drawing attention in recent years (Liu et al., 2018). MVs derived from Gram-positive bacteria are similarly sized (50 to $150 \mathrm{~nm}$ in diameter) and are rich in membrane lipids as well as toxins (Deatherage \& Cookson, 2012). Microscopy of Gram-positive bacteria including Bacillus subtilis, Staphylococcus aureus, and Streptococcus pneumoniae showed the budding events and protein localization in MVs (Liu et al., 2018).

Transmission electron microscopy of $S$. aureus revealed MVs released from the cell surface (Fig. 3A) (Wang et al., 2018). MVs pelleted after centrifugation were spherical and approximately $100 \mathrm{~nm}$ in diameter (Fig. 3B). Since Gram-positive bacteria have a thick cell wall that consists of peptidoglycan, how MVs are released is not well understood (Toyofuku et al., 2015).

\section{ARCHAEAL MVs}

Belonging to the third domain of life on Earth, archaea are prokaryotic single-cell organisms. Archaeal MVs range from 90 to $230 \mathrm{~nm}$ in diameter and contain membrane lipids and surface (S)-layer proteins also derived from the archaeal cell surface (Deatherage \& Cookson, 2012). Thermococcus species produce MVs by a budding process from the cell envelope, similar to ectosome formation in eukaryotic cells (Marguet et al., 2013). Strains of Pyrococcus and Thermococcus form various types of spherical MVs and unusual filamentous struc- tures (Soler et al., 2008).

Transmission electron microscopy revealed MVs of Sulfolobus solfataricus (Fig. 4) (Ellen et al., 2009). These vesicles showed the proteinaceous S-layer presumably derived from the archaeal species. Archaeal MVs associated with DNA could be confused with virions in environmental studies (Marguet et al., 2013).

\section{FUNGAL EXOSOMES}

The baker's yeast Saccharomyces cerevisiae is the most representative yeast widely used in a variety of food industry and biotechnology. The fungal species is known to form exosomes (Joffe et al., 2016). Transmission electron microscopy revealed bilayered structures with varying electron density (Fig. 5) (Oliveira et al., 2010). They are overall round or ovoid and

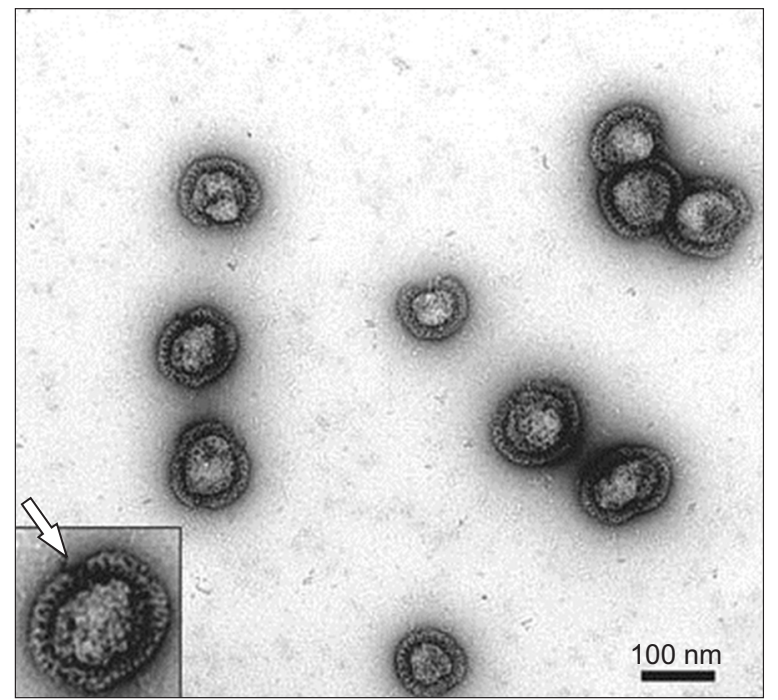

Fig. 4. Transmission electron micrograph of membrane vesicles of Sulfolobus solfataricus. These vesicles are surrounded with surface-layer (arrow). From Ellen et al., 2009 with permission from the publisher.
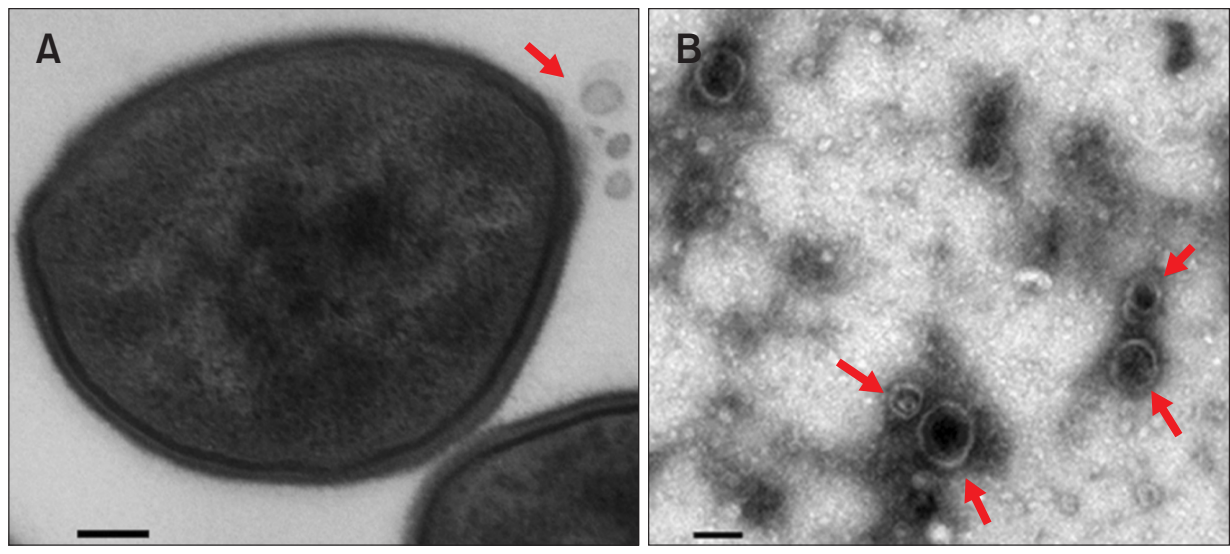

Fig. 3. Transmission electron micrographs of membrane vesicles of Staphylococcus aureus. (A) Membrane vesicles (arrow) released from the cell. (B) Membrane vesicles pelleted by ultracentrifugation from the bacterial culture. Bars $=100 \mathrm{~nm}$. A and B: from Wang et al., 2018 with permission from the publisher. 


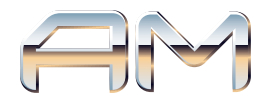

approximately $100 \mathrm{~nm}$ in diameter. The mechanisms of exosome release across the complex molecular network of the fungal cell wall remain still unclear (Joffe et al., 2016).

Furthermore, filamentous fungi also produce exosomes. Scanning electron microscopy of Alternaria infectoria revealed exosomes on the surface hyphae (Silva et al., 2014). Transmission electron microscopy showed membrane-associated electron-dense vesicles, heavily pigmented vesicles, and electron-dense vesicles (Silva et al., 2014). These vesicles are approximately $20 \mathrm{~nm}$ in diameter.

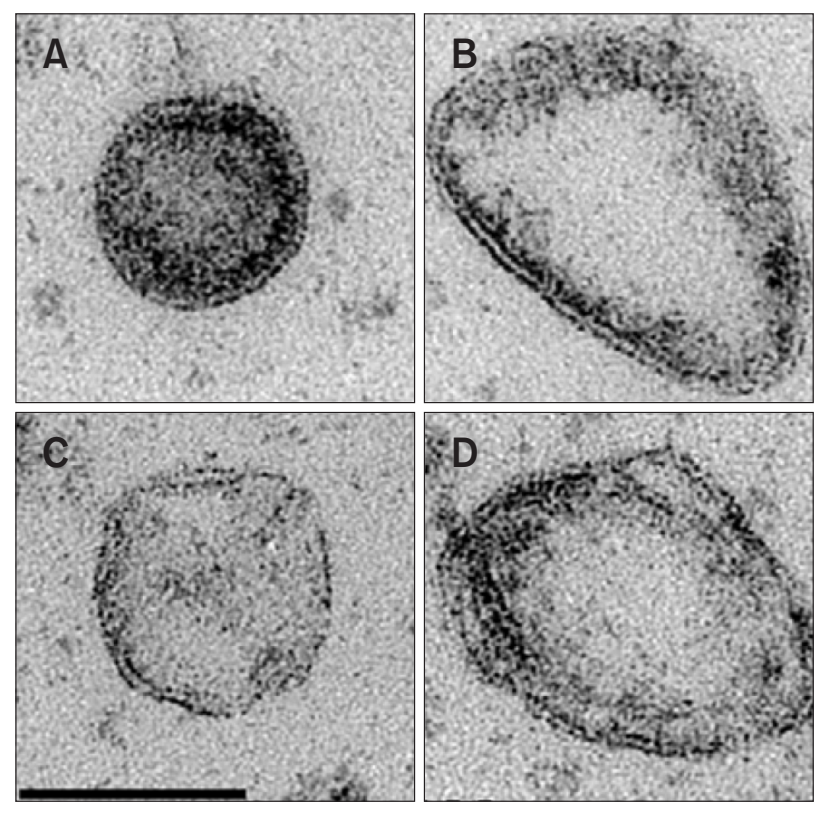

Fig. 5. Transmission electron micrographs of membrane vesicles of Saccharomyces cerevisiae. (A-D) Exosomes. They are bilayered structures with varying electron density. Bar=100 nm. A-D: from Oliveira et al., 2010 with permission from the publisher.

\section{INTRACELLULAR VESICLES AND MINICELLS}

Besides extracellular vesicles, bacteria unexpectedly form intracellular vesicles. While no standard set of membranous organelles is present in prokaryotes, some bacterial species can possess membrane-bound compartments within the cells, creating distinct microenvironments for a given task (Giessen \& Silver, 2016). Transmission electron microscopy of Agrobacterium tumefaciens revealed membrane-bound structures with $100 \mathrm{~nm}$ in diameter (Fig. 6) (Seufferheld et al., 2011). These vesicles are called acidocalcisomes, volutin or polyphosphate granules composed of phosphorus as polyphosphate and pyrophosphate as well as calcium (Giessen \& Silver, 2016). They appear partially filled or empty vacuoles due to the chemical fixation protocol that may facilitate diffusion of the electron-dense material to the outside (Seufferheld et al., 2011).

Small spherical cells, referred to as minicells, are produced from a strain of E. coli (Martinez-Penafiel et al., 2012). The strain harboring the prophage mEp021 displayed a variety of abnormal phenotypes such as desiccated-like bacilli, minicells, and vesicles. Although similar to MVs in shape, minicells appear as rosary-like structures and are approximately $400 \mathrm{~nm}$ in diameter (Martinez-Penafiel et al., 2012).

\section{MOLECULAR COMPOSITION OF MVs AND FUNGAL EXOSOMES}

Given their formation from cell surfaces, it is natural to state that MVs contain many components of the parent cell (Bitto et al., 2017). In addition to membrane proteins, DNA, toxins, and signaling molecules can be incorporated into the membrane or lumen of the MV (Deatherage \& Cookson, 2012). To confirm the presence of lipid and DNA, MVs of Pseudomonas aeruginosa were stained with the lipophilic fluorescent dye

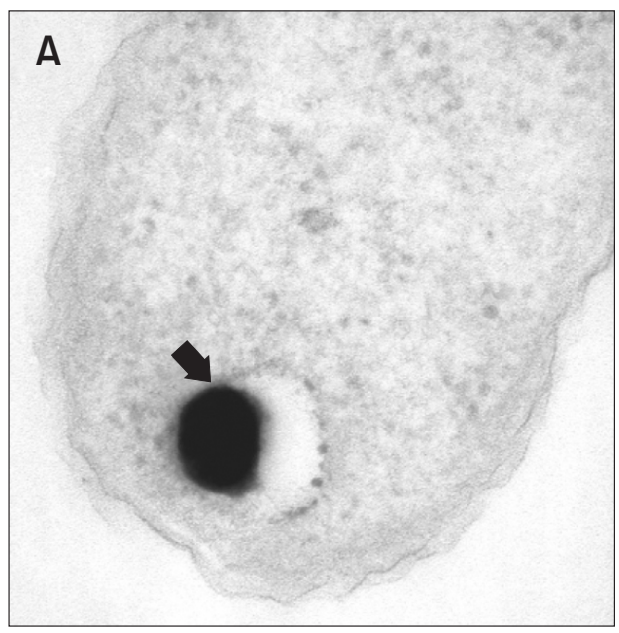

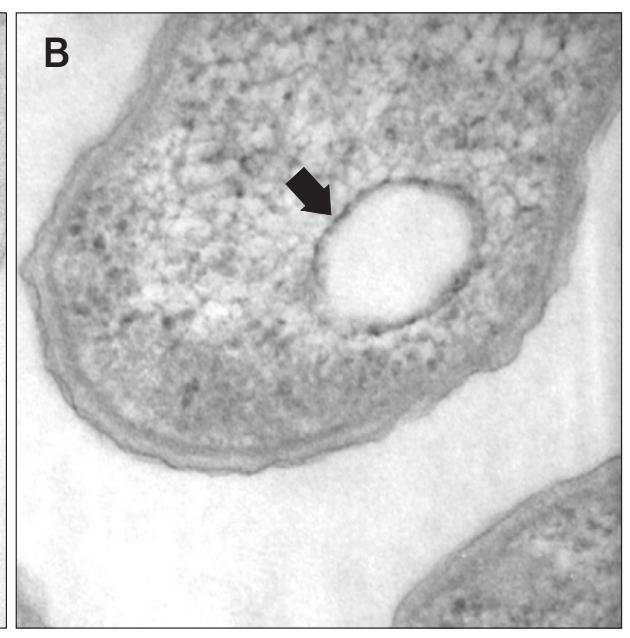

Fig. 6. Transmission electron micrographs of Agrobacterium tumefaciens. (A) Cell having the partially filled acidocalcisome (arrow). (B) Cell having the empty acidocalcisome (arrow). A and B: from Seufferheld et al., 2011 with permission from the publisher. 

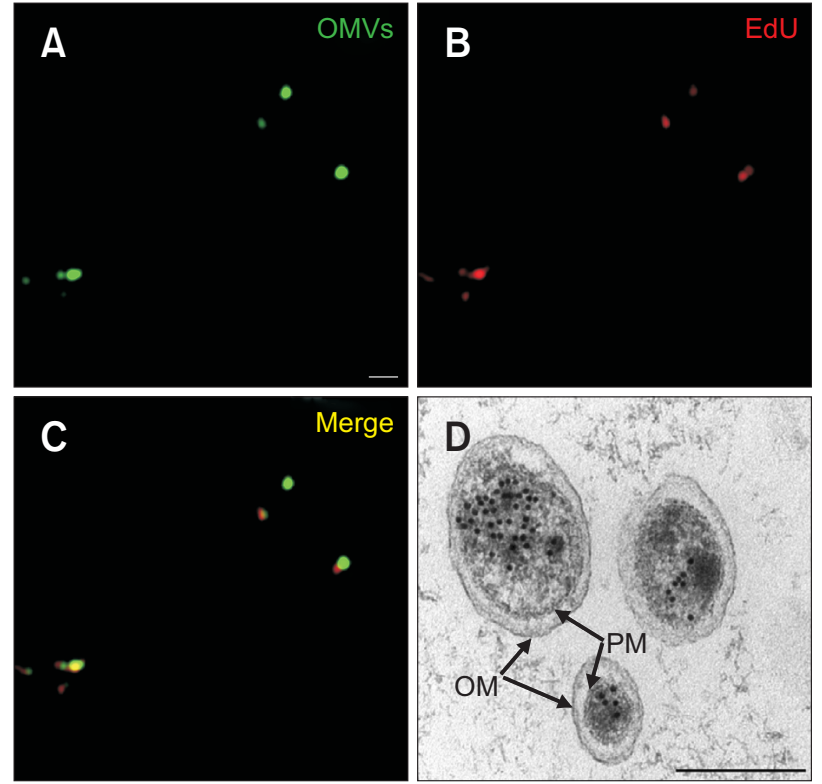

Fig. 7. Localization of DNA in membrane vesicles. (A-C) Super resolution micrographs of membrane vesicles of Pseudomonas aeruginosa. (A) Membrane vesicles stained with the lipophilic fluorescent dye, DiO (green). Bar=1 $\mu \mathrm{m}$. (B) Membrane vesicles stained with the DNA-specific dye, 5- ethynyl-2-deoxuridine (EdU). (C) Merged image of (A) and (B). (D) Transmission electron micrograph of membrane vesicles of Shewanella vesiculosa. Electron-dense particles indicate gold particles with an antibody specific for DNA. OM, outer membrane; PM, plasma membrane. A-C: from Bitto et al., 2017; D: from Pérez-Cruz et al., 2013 with permission from the publisher.

DiO and the DNA-specific dye [5- ethynyl-2-deoxuridine (EdU)] (Bitto et al., 2017). Super resolution microscopy revealed the localization of lipid and DNA in the DiO and EdUstained MVs, respectively (Fig. 7A and B). The merged image showed the colocalization of these macromolecules (Fig. 7C). Immunogold labeling was performed to localize DNA in the
MVs of Shewanella vesiculosa (Pérez-Cruz et al., 2013). Electron-dense gold particles with an antibody specific for DNA were detected in the vesicles (Fig. 7D).

To check if MVs contain RNA, MVs of Vibrio cholerae were disrupted and treated with RNA extraction and RNase (Sjöström et al., 2015). No RNA was detected from the RNasetreated sample, implying that the MVs harbor RNA. Exosomes of Cryptococcus neoformans and S. cerevisiae also contain RNA, suggesting that exosomes may be determinants for various biological processes such as intercellular communication and pathogenesis (De Silva et al., 2015).

\section{CONCLUSIONS}

Microbes including bacteria, archaea, and fungi release nanosized membrane-bound structures into their extracellular environment. These extracellular vesicles have been rigorously investigated using a variety of microscopes. A variety of membrane remodeling proteins are involved in the biological process (Bohuszewicz et al., 2016). Since these vesicles are derived from the cell envelope, it is crucial to employ in situ technique for a better understanding of the cellular process occurring in nature. Electron cryo-tomography and subtomogram averaging can be used to visualize the details of the vesicle genesis and release at a near-native state. Elucidation of the nature of microbial extracellular vesicles will provide novel insights into the understanding of fundamental biological processes such as gene transfer, biofilm formation, pathogenicity, and survival in nature.

\section{CONFLICT OF INTEREST}

No potential conflict of interest relevant to this article was reported.

\section{REFERENCES}

Bielaszewska M, Ruter C, Bauwens A, Greune L, Jarosch K A, Steil D, Zhang W, He X, Lloubes R, Fruth A, Kim K S, Schmidt M A, Dobrindt U, Mellmann A, and Karch H (2017) Host cell interactions of outer membrane vesicle-associated virulence factors of enterohemorrhagic Escherichia coli 0157: intercellular delivery, trafficking and mechanisms of cell injury. PLoS Pathog. 13, e1006159.

Bitto N J, Chapman R, Pidot S, Costin A, Lo C, Choi J, D'Cruze T, Reynolds E C, Dashper S G, Turnbull L, Whitchurch C B, Stinear T P, Stacey K J, and Ferrero R L (2017) Bacterial membrane vesicles transport their DNA cargo into host cells. Sci. Rep. 7, 7072.

Bohuszewicz O, Liu J, and Low H H (2016) Membrane remodeling in bacteria. J. Struct. Biol. 196, 3-14.

Chernov V M, Mouzykantov A A, Baranova N B, Medvedeva E S, Grygorieva T Y, Trushin M V, Vishnyakov I E, Sabantsev A V, Borchsenius S N, and Chernova O A (2014) Extracellular membrane vesicles secreted by mycoplasma Acholeplasma laidlawii PG8 are enriched in virulence proteins. J. Proteom. 110, 117-128.

De Silva R P, Puccia R, Rodrigues M L, Oliveira D L, Joffe L S, Cesar G V, Nimrichter L, Goldenberg S, and Alves L R (2015) Extracellular vesicle-mediated export of fungal RNA. Sci. Rep. 5, 7763.

Deatherage B L and Cookson B T (2012) Membrane vesicle release in bacteria. eukaryotes, and archaea: a conserved yet underappreciated aspect of microbial life. Inf. Immun. 80, 1948-1957.

Ellen A F, Albers S-V, Huibers W, Pitcher A, Hobel C F V, Schwarz H, Folea M, Schouten S, Boekema E J, Poolman B, and Driessen A J M (2009) Proteomic analysis of secreted membrane vesicles of archaeal Sulfolobus species reveals the presence of endosome sorting complex components. Extremophiles 13, 67-79. 
Giessen T W and Silver P A (2016) Encapsulation as a strategy for the design of biological compartmentalization. J. Mol. Biol. 428, 916-927.

Ionescu M, Zaini P A, Baccari C, Tran S, Da Silva A M, and Lindow S E (2014) Xylella fastidiosa outer membrane vesicles modulate plant colonization by blocking attachment to surfaces. PNAS 111, E39103918.

Joffe L S, Nimrichter L, Rodrigues M, and Del Poeta M (2016) Potential roles of fungal extracellular vesicles during infection. $m$ Sphere $\mathbf{1}$, e00099-16.

Knox K W, Vesk M, and Work E (1966) Relation between excreted lipopolysaccharide complexes and surface structures of a lysine-limited culture of Escherichia coli. J. Bacteriol. 92, 1206-1217.

Liu Y, Defourny K A Y, Smid E J, and Abee T (2018) Gram-positive bacterial extracelluar vesicles and their impact on health and disease. Front. Microbiol. 9, 1502.

Marguet E, Gaudin M, Gauliard E, Fourquaux I, Le Blond du Plouy S, Matsui I, and Forterre P (2013) Membrane vesicles, nanopods and/ or nanotubes produced by hyperthermophilic archaea of the genus Thermococcus. Biochem. Soc. Trans. 41, 436-442.

Martínez-Penafiel E, Fernandez-Ramirez F, Ishida C, Reyes-Cortes R, Sepulveda-Robles O, Guarneros-Pena G, Bermudez-Cruz R M, and Kameyama L (2012) Overexpression of Ipe protein from the coliphage mEp021 induces pleiotropic effects involving haemolysis by HlyE-containing vesicles and cell death. Biochimie 94, 1262-1273.

Oliveira D L, Nakayasu E S, Joffe L S, Guimaraes A J, Sobreira T J P, Nosanchuk J D, Cordero R J B, Frases S, Casadevall A, Almeida I C, Nimrichter L, and Rodrigues M L (2010) Characterization of yeast extracellular vesicles: evidence for the participation of different pathways of cellular traffic in vesicle biogenesis. PLOS ONE 5, e11113.

Pérez-Cruz C, Carrión O, Delgado L, Martinez G, López-Iglesias C, and Mercade E (2013) New type of outer membrane vesicle produced by the Gram-negaive bacterium Shewanella vesiculosa $\mathrm{M}^{\top}$ : implications for DNA content. Appl. Environ. Microbiol. 79, 1874-1881.

Pérez-Cruz C, Delgado L, López-Iglesias C, and Mercade E (2015) Outerinner membrane vesicles naturally secreted by Gram-negative pathogenic bacteria. PLoS ONE 10, e0116896.

Raposo G and Stoorvogel W (2013) Extracellular vesicles: exosomes, microvesicles, and friends. J. Cell Biol. 200, 373-383.

Schwechheimer C and Kuehn M J (2015) Outer-membrane vesicles from Gram-negative bacteria: biogenesis and functions. Nat. Rev. Microbiol. 13, 605-619.

Seufferheld M J, Kim K M, Whitfield J, Valerio A, and Caetano-Anolles G (2011) Evolution of vacuolar proton pyrophosphatase domains and volutin granules: clues into the early evolutionalry origin of the acidocalcisome. Biol. Direct 6, 50.

Silva B M A, Prados-Rosales R, Espadas-Moreno J, Wolf J M, LuqueGarcia J L, Goncalves T, and Casadevall A (2014) Characterization of Alternaria infectoria extracelluar vesicles. Med. Mycol. 52, 202-210.

Sjöström A E, Sandblad L, Uhlin B E, and Wai S N (2015) Membrane vesicle-mediated release of bacterial RNA. Sci. Rep. 5, 15329.

Soler N, Marguet E, Verbavatz J-M, and Forterre P (2008) Virus-like vesicles and extracellular DNA produced by hyperthermophilic archaea of the order Thermococcales. Res. Microbiol. 159, 390-399.

Thery C, Ostrowski M, and Segura E (2009) Membrane vesicles as conveyors of immune responses. Nat. Rev. Immunol. 9, 581-593.

Toyofuku M, Tashiro Y, Hasegawa Y, Kurosawa M, and Nomura N (2015) Bacterial membrane vesicles, an overlooked environmental colloid: biology, environmental perspectives and applications. Adv. Colloid Interface Sci. 226, 65-77.

Wang X, Thompson C D, Weidenmaier C, and Lee J C (2018) Release of Staphyloccous aureus extracellular vesicles and their application as a vaccine platform. Nat. Commun. 9, 1379. 